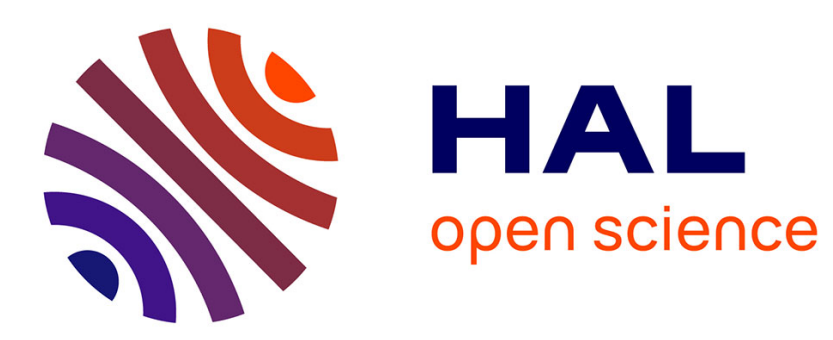

\title{
Justice environnementale et approche par les capabilités
}

Jérôme Ballet, Damien Bazin, Jérôme Pelenc

\section{- To cite this version:}

Jérôme Ballet, Damien Bazin, Jérôme Pelenc. Justice environnementale et approche par les capabilités.

2013. halshs-01071203

\section{HAL Id: halshs-01071203 \\ https://shs.hal.science/halshs-01071203}

Preprint submitted on 3 Oct 2014

HAL is a multi-disciplinary open access archive for the deposit and dissemination of scientific research documents, whether they are published or not. The documents may come from teaching and research institutions in France or abroad, or from public or private research centers.
L'archive ouverte pluridisciplinaire HAL, est destinée au dépôt et à la diffusion de documents scientifiques de niveau recherche, publiés ou non, émanant des établissements d'enseignement et de recherche français ou étrangers, des laboratoires publics ou privés. 


\section{Justice environnementale et approche par les capabilités}

Jérôme Ballet ${ }^{\text {a) }}$ Damien Bazin ${ }^{\text {b) }}$, Jérôme Pelenc ${ }^{\text {c) }}$.

Auteur de correspondance : Jérôme Ballet

Résumé : Nous montrons que l'approche par les capabilités offre de sérieuses pistes pour donner tout son sens à la justice environnementale abordée sous l'angle de la justice comparative. L'approche des capabilités permet de prendre en compte les trois enjeux que soulève la justice environnementale : la distribution des bénéfices et des nuisances liés à l'environnement, les enjeux de participation des populations dans l'élaboration des régulations environnementales, la reconnaissance des identités collectives. Cependant, cela suppose d'une part de donner une priorité à la liberté de processus sur la liberté d'opportunités, d'autre part l'introduction du concept de capabilité collective.

Mot clés : Capabilités, justice comparative, justice environnementale, justice transcendantale.

Abstract: We show that the capability approach offers serious tracks to give full meaning to environmental justice from the perspective of comparative justice. The capability approach allows to take into account the three issues raised by environmental justice: the distribution of benefits and nuisances related to the environment, issues of people participation in the development of environmental regulations, recognition of collective identities. However, this requires on one hand to give a priority to freedom of process over freedom of opportunities, and on other hand the introduction of the concept of collective capability.

Keywords: Capabilities, comparative justice, environmental justice, transcendental justice

Classification JEL : O10, Q56, Z1.

\footnotetext{
${ }^{1}$ a) UMI Résiliences (Institut de Recherche pour le Développement) 32 avenue Henri Varagnat, 93143 Bondy ; Fonds pour la Recherche en Ethique Economique (FREE) \& chercheur associé au Gretha-Université de Bordeaux.jballetfr@yahoo.fr

b) Université de Nice Sophia Antipolis, UFR Institut Supérieur d'Economie et Management (ISEM). Centre National de la Recherche Scientifique, Groupe de Recherche en Droit, Economie et Gestion (GREDEG) UMR 7321 UNS-CNRS, 250, rue Albert Einstein, 06560 Valbonne - France. damien.bazin@gredeg.cnrs.fr

c) Centre de Recherche et de Documentation sur les Amériques (CREDA), 28 rue Saint-Guillaume, 75007 Paris. Jerome.pelenc@gmail.com
} 


\section{Justice environnementale et approche par les capabilités}

\section{Introduction}

Les enjeux éthiques liés à l'environnement se sont historiquement scindés en deux approches distinctes : d'un côté l'éthique environnementale et de l'autre la justice environnementale. A la suite des travaux pionniers de Leopold (1949), la question de l'éthique environnementale s'est principalement focalisée sur la relation de l'homme à la nature. Il s'agissait globalement de remettre en cause la vision anthropocentriste de l'humain dominant la nature. Naess (1973) en fondant la Deep Ecology (écologie profonde), puis des auteurs comme Passmore (1974), Stone (1974), Rolston (1975) pour n'en citer que quelques-uns, ont souligné avec vigueur l'importance de la vie non humaine et le respect que l'homme doit lui accorder. En se focalisant uniquement sur la relation de l'homme à la nature, cette perspective a laissé de côté les relations entre les hommes. Or ces dernières ne sont pas exemptes de médiation par la nature. Comme le soulignent Wapner et Matthew (2009), il faut aussi se demander comment les êtres humains se traitent ou se maltraitent les uns les autres à travers l'usage de la nature. La nature joue un rôle parce qu'elle est non seulement un espace de vie des individus (que l'on peut apprécier soit d'un point de vue biocentré, soit anthropocentré), mais elle est aussi un moyen de médiation entre les individus. Elle est un support essentiel des relations des hommes entre eux. A travers cette médiation, une image de la manière dont les hommes se traitent ou se maltraitent surgit. En opposition à l'éthique environnementale, la justice environnementale s'interroge sur les relations que les hommes ont entre eux par le biais de l'environnement. Plus particulièrement, elle met en évidence les disparités en matière d'accès aux ressources naturelles et de risques liés aux pollutions.

Cet article défend l'idée d'une justice environnementale appréhendée à partir d'une approche comparative de la justice. Dans ce cadre, l'approche des capabilités nous aide à fournir une analyse pertinente des injustices. Cependant, cette dernière doit faire l'objet d'un aménagement visant à prendre en compte, dans le jugement d'évaluation des sociétés, les capabilités collectives. Dans une première section nous caractérisons la justice environnementale. Nous faisons valoir en particulier qu'elle s'articule autour de trois principes: l'équité, la reconnaissance et la participation. Dans une seconde section, reconnaissant la justice environnementale comme un ensemble de mouvements cohérents avec une approche comparative de la justice, nous synthétisons le débat récent sur l'opposition entre justice transcendantale et justice comparative. Nous situons à sa "juste » place la justice comparative par rapport à la justice transcendantale. Enfin, dans une troisième section nous défendons l'idée que l'approche des capabilités constitue une métrique appropriée pour une approche de la justice comparative appliquée à la justice environnementale. Cela suppose néanmoins d'étendre l'approche des capabilités à la prise en compte, dans le jugement d'évaluation des sociétés, des capabilités collectives.

\section{Sur la notion de justice environnementale}

La justice environnementale ne repose pas sur une définition unique et consensuelle. Elle renvoie à un ensemble hétérogène de préoccupations dont on trouve les origines dans les mouvements issus de la société civile. Elle se focalise initialement sur deux phénomènes : d'une part, les mouvements anti-toxicités de la pollution et d'autre part les mouvements contre le racisme environnemental.

\subsection{De l'hétérogénéité des mouvements à l'unité des préoccupations}


Le premier mouvement a pris de l'ampleur avec le cas Love canal $^{2}$ aux USA en 1978. Une décharge toxique de plus de 20000 tonnes de déchets chimiques a été découverte enfouie sous une école primaire et dans le quartier résidentiel de la classe ouvrière de LaSalle à Niagara Falls, dans l'Etat de New York. Les effets sur la santé pour les résidents ont été alarmants, avec une incidence élevée de cancer, de fausses couches, de maladies rares et d'anomalies congénitales. Si ce cas fût emblématique aux USA, Pellow (2000) et Melosi (2004) relèvent que les stations d'épuration des eaux usées et les décharges municipales ont depuis des temps anciens été localisées proches des zones d'habitation des populations ouvrières et des groupes minoritaires, ce qui accroît très fortement les risques de contamination par les pollutions pour ces populations. Ce premier mouvement insiste sur le cumul des inégalités socio-économiques et des inégalités écologiques, plus spécifiquement la charge des risques liés aux pollutions.

Le second mouvement débute avec la protestation aux USA en 1982 contre un nouveau site d'enfouissement de déchets toxiques à Warren en Caroline du Nord. Or Warren était non seulement une commune pauvre mais aussi composée à $65 \%$ d'afro-américains. Cette situation fût une des premières où les mouvements pour les droits des citoyens et les groupes écologistes menèrent une action commune. Ce mouvement a souligné que les dangers liés à la pollution touchaient particulièrement les populations noires et asiatiques des USA (Bryant et Mohai, 1982). Ce second mouvement a lui insisté sur la notion de racisme environnemental, i.e. le fait que ce sont les populations de couleur qui sont plus spécifiquement affectés par les risques de pollution.

Si cette double origine de la notion de justice environnementale a donné lieu à des débats pour déterminer s'il s'agissait de problèmes spécifiques à une classe sociale (ouvriers) ou à des minorités (afro-américains, latino-américains, asiatiques, etc.), les mouvements pour la justice environnementale procèdent en fait de la convergence de multiples préoccupations, parmi lesquelles le mouvement des droits des citoyens, le mouvement pour la santé et la sécurité au travail, le mouvement pour les droits des populations autochtones, le mouvement pour la sécurité et la santé publique, etc. (Faber et McCarthy, 2003). Une telle hétérogénéité peut faire craindre à une dilution du propos, voire à une confusion (Getches et Pellow, 2002), mais derrière les divergences, se profile la recherche d'une unité d'un mouvement large autour de différents éléments qui composent la justice (Epstein, 1997, Faber et Mc Carthy, 2003, Schlosberg, 2003). Bien sûr, les revendications en faveur de la justice environnementale ne se sont pas cantonner aux USA. Elles ont connu des progressions un peu partout dans le monde. Nous ne cherchons pas à dresser les portraits de ces mouvements à travers le monde (cf. Schlosberg, 2007 pour une première approche). Nous souhaitons seulement faire ressortir les enjeux qui président à leurs actions.

Schlosberg (2007) note que la notion de justice environnementale s'articule autour de trois enjeux principaux : la distribution, la participation et la reconnaissance. Ces trois enjeux s'articulent l'un à l'autre de manière complémentaire. L'absence de reconnaissance des populations joue un rôle important dans la distribution inéquitable des bénéfices environnementaux, comme dans l'exposition aux pollutions. A son tour la participation des populations au processus de décision concernant les enjeux environnementaux ne se réalise

\footnotetext{
${ }^{2}$ Cette appellation n'a rien de romantique. Bien au contraire, à la fin XIX ${ }^{\text {ième }}$ siècle aux USA, un entrepreneur du nom de William T. Love, ordonna la construction (inachevée) d'un canal de plus d'un kilomètre de long. Le canal fut racheté dans les années quarante par une firme américaine Hooker Chemical devenue depuis 1'Occidental Petroleum Corporation (Oxy). La compagnie y a déversée en toute discrétion plus de 20.000 tonnes de déchets toxiques causant ainsi une infiltration de produits chimiques dans le sous-sol. Cette zone est depuis sinistrée.
} 
que si elles sont reconnues comme acteur à part entière. Nous mettons en évidence cette articulation.

\subsection{Les trois enjeux de la justice environnementale}

L'inéquité dans la distribution est au fondement de la justice environnementale. Les travaux relatifs à ce champ d'analyse soulignent que les coûts des pollutions comme les bénéficies de la protection de l'environnement sont inégalement répartis à travers la société (Edwards, 1995). Les analyses s'appuient sur des constats multiples concernant, d'une part les coûts disproportionnés subis par certaines catégories de populations, tels que les localisations de sites de déchets dangereux, des incinérateurs et des décharges, les localisations des industries polluantes, les proportions de travailleurs dans les activités dangereuses, mais aussi les différences et la concentration de toxicité dans la consommation tels que les poissons contaminés ; et d'autre part les inégalités dans les bénéfices de biens environnementaux tels que les parcs, les aires naturelles de jeux, la qualité de l'eau et de l'air, etc. (pour une collection d'analyses voir par exemple Agyeman et al., 2003). Mais il ne s'agit pas de réduire les questions d'inéquité à des constats d'inégalités entre individus. Ce sont souvent les communautés dans leur ensemble qui sont affectées et pas les individus pris un à un. Schlosberg (2007) note par exemple que les compagnies minières qui exploitent des gisements d'uranium situés sur le territoire de la communauté Navajo d'Arizona du nord, une des plus grandes communautés autochtones des USA (environ 200000 individus), embauchent ces derniers à un taux de salaire plus bas que le standard national et ne mettent pas en œuvre les standards de sécurité avec autant de rigueur sur ces sites qu'ailleurs aux USA. Il en découle une proportion de cancer chez les jeunes dans cette population dix sept fois supérieur à la moyenne nationale. LaDuke (2002) dresse un constat aussi dramatique pour la tribu Shoshone dans le Nevada dont le territoire sert d'entreposage pour les déchets radioactifs. Les inégalités ne peuvent, dans ce genre de cas, être réduites aux individus puisque c'est bien la communauté dans son ensemble qui est affectée et qui supporte le poids des risques environnementaux. Elle est également atteinte dans son identité et les actions et demandes en faveur d'une justice environnementale ne sont pas portées par des individus séparés mais par des communautés et leurs représentants (Getches et Pellow, 2002). Pour cette raison, les mouvements environnementaux n'utilisent d'ailleurs guère la notion d'équité mais préfèrent celle de justice qui ne se résout pas dans cette première (Taylor, 2000). Elle renvoie également à la notion de participation. En effet, si les individus peuvent être traités équitablement de manière passive, les mouvements pour la justice environnementale insistent sur la dimension participative de la justice.

Au-delà du contenu des décisions, c'est donc l'inéquité dans le processus qui est dénoncé, ce qui explique que les premiers mouvements, à l'image du First National People of Color Environmental Justice Leadership Conference, ont insisté sur la justice procédurale et la participation de manière équitable des minorités au processus de décision (Freudenberg et Steinsapir, 1992; Scholsberg, 1999). Les revendications de participation émanant des mouvements pour la justice environnementale s'articulent autour de trois exigences (Schlosberg, 2007). Premièrement, les mouvements demandent que les populations soient informées des enjeux et des risques qui les concernent. L'information doit être fournie par les entités administratives et les industries de manière transparente. Tandis que les populations sont jugées habituellement incompétentes pour comprendre les questions techniques, la première revendication porte précisément sur la mise à disposition des informations. Deuxièmement, les mouvements réclament que les populations soient parties prenantes du processus de décision et que leur opinion soit considérée avec autant de poids que celle des autres parties du processus de décision. Enfin, troisièmement, ils demandent que les 
populations soient impliquées dans le processus de recherche sur les effets des projets aux côtés des scientifiques. Il s'agit de faire valoir les connaissances des populations au-delà des compétences scientifiques puisque bien souvent les cas tel celui de Love canal ont pu être défendus grâce à l'ensemble des effets ressentis par les populations et qu'elles pouvaient décrire.

Cependant, pour obtenir une justice procédurale, la reconnaissance des populations est un préalable. Elle est la base de la possibilité d'exprimer non seulement son opinion mais aussi et surtout de voir son avis pris en compte. Dans l'affaire Love canal, Gibbs (1982) note que les femmes qui tentaient de s'exprimer pour faire valoir leur point de vue et rapporter leur témoignage étaient systématiquement ignorées, discréditées parce que considérées comme irrationnelles, émotives et mal informées. Hamilton (1994) dresse le même constat au travers d'un cas de projet d'incinérateur à Los Angeles. Les femmes et les populations issues des minorités sont systématiquement dénigrées par les autorités car jugées irrationnelles et mal informées. Il s'agit là d'un problème d'autant plus marqué que les populations issues des minorités ne sont historiquement pas présentes dans les mouvements environnementalistes (Bullard, 1993). Elles n'ont pas pu acquérir de crédibilité par ce biais. Les mouvements pour la justice environnementale visent par conséquent à donner une voix à ces populations. Ils contribuent à leur accorder un poids politique, à favoriser leur empowerment (Bullard, 1993) ou leur agencéité (Di Chiro, 1992) en insistant sur leur reconnaissance.

Si la reconnaissance est à la base de la justice procédurale, son absence est aussi à l'origine de nombreuses inéquités. LaDuke (2002) souligne à cet égard que certains standards de pollution sont fondés sur des règles qui ignorent les spécificités culturelles des populations par défaut de reconnaissance de celles-ci. Par exemple, il soutient que le niveau de rejet de dioxine de l'industrie de la papeterie dans les rivières et fleuves est défini aux USA par un taux supportable pour la population au regard de la consommation de poissons issus des ces fleuves et rivières. Mais ce standard se fonde sur une consommation moyenne de poissons d'eau douce dans la population ; critère totalement inapproprié pour les populations qui en font une grande consommation, comme c'est le cas des populations autochtones indiennes. Dans un autre registre, Schlosberg (2007) souligne que la décision du Service des forêts des USA d'autoriser l'usage d'eaux usées traitées pour accroître l'enneigement à fin de développer les activités de sports d'hiver sur des montagnes locales considérées comme sacrées par les treize tribus indiennes n'a pas tenu compte de l'importance de ces montagnes pour ces populations parce que leurs spécificités culturelles n'ont pas été reconnues. Il ne s'agit pas dans ces cas uniquement de reconnaissances individuelles mais bien d'identité collective. Ce sont les populations en tant que groupes ou communautés avec leurs propres spécificités qui ne sont pas reconnus (Agyeman et al., 2003). Comme le souligne Pulido (1996), la spécificité des mouvements en faveur de la justice environnementale, en comparaison des autres organisations environnementales, consiste à dresser les contours des populations en tant que communautés c'est à dire en tant qu'entité spatiale qui peut être caractérisée par une appartenance (par exemple: appartenance de classe, appartenance ethnique, etc.). Il s'agit avant tout d'un mouvement pour la reconnaissance des communautés dont les enjeux sont environnementaux sur un territoire particulier. Les injustices environnementales sont aussi des atteintes à l'intégrité, certes physique, mais également identitaire. Elles sont assimilées par les communautés affectées à une forme de "génocide » (Krauss, 1994 ; Bretting et Prindeville, 1998). Les motifs d'engagement dans les mouvements activistes sont d'ailleurs autant ceux concernant la santé publique que la préservation de la culture (Prindeville, 2004). Pour cette raison, une politique de défense des communautés consiste en la création de fondations, à l'image de la fondation White Earth Land Recovery ${ }^{3}$

\footnotetext{
${ }^{3}$ Dont le slogan est: «Facilitating the recovery of the original land base of the White Earth Reservation». http://welrp.org/
} 
qui rachètent les terres pour les réallouer aux populations autochtones, ce qui leur confère un pouvoir collectif et une reconnaissance (LaDuke, 2002).

La justice environnementale articule par conséquent l'inéquité, la participation et la reconnaissance ; avec en son cœur la reconnaissance puisque d'une part le manque de reconnaissance est analysé comme la cause fondamentale de l'inéquité et, d'autre part, elle constitue la condition d'une réelle participation. A travers cette démarche, les mouvements en faveur d'une plus grande justice environnementale militent pour une réduction des injustices, sans poser en toile de fonds de définition de ce qu'est une société parfaitement juste. L'approche de ces mouvements renvoie au débat actuel sur l'opposition entre justice comparative et justice transcendantale.

\section{Justice comparative versus justice transcendantale}

Schlosberg (2007) note que la plupart de la littérature sur la justice environnementale examine des cas particuliers qui relatent des histoires d'injustice, explorent les arguments concernant le caractère intentionnel ou raciste des ces injustices, et analysent les manières d'opérationnaliser et mesurer ces injustices. Un tel point de vue semble en cohérence avec la notion de justice comparative défendue par Sen (2006a, 2009).

\subsection{De l'opposition entre conception comparative et conception transcendantale de la justice...}

Dans son ouvrage Idées de justice, Sen (2009), poursuivant une réflexion entamée dans un article de 2006 intitulé What do we want from a theory of justice?, oppose une approche transcendantale de la justice et une approche comparative. Il développe ainsi une réflexion sur la réduction des injustices dans des contextes où ce qui compte est ce que vivent et ressentent les gens. Cette conception de la justice implique, au regard de certaines expériences historiques d'injustice mondialement reconnues, qu'il est important de comprendre que les populations cherchent avant tout à supprimer les injustices flagrantes dans la mesure de leurs capacités, plutôt qu'à chercher un monde parfaitement juste. Cela semble indiquer que l'action sociale d'une personne ou d'un groupe soumis à une injustice, vise à sortir de l'injustice ; ce qui n'équivaut certainement pas à entrer dans une situation de justice parfaite. Sen (2009) identifie trois caractéristiques majeures d'une justice comparative par rapport à une justice transcendantale. Tout d'abord, tandis que la justice transcendantale s'attache à définir ce qu'est une société parfaitement juste, la justice comparative doit permettre d'identifier les moyens qui réduiront les situations d'injustice. Ensuite, la justice transcendantale se focalise sur les institutions justes qui doivent gouverner la société. La justice comparative, sans nier le rôle notable des institutions, tient compte des comportements de transgression des individus et pas seulement des insuffisances institutionnelles. Elle se centre sur la vie réelle des gens et non sur la vie qu'il devrait mener dans une société avec des institutions justes. Enfin, alors que la justice transcendantale cherche à définir un état unique qui caractérise une société juste, la justice comparative admet le pluralisme des valeurs. Ce pluralisme ne signifie pas relativisme, mais d'une part que les nombreux problèmes relatifs à la justice doivent faire l'objet d'un débat argumenté, qui peut dans certains cas aboutir à un consensus; d'autre part que l'absence de consensus ne découle pas d'un défaut de raisonnement et de réflexion, mais au contraire de priorités rivales qui ont franchi le crible de la raison. En somme la justice comparative est plus orientée pratiquement que la justice transcendantale.

\section{2. ... à la complémentarité}


En défendant une approche comparative de la justice, Sen (2009) s'oppose particulièrement à la théorie de la justice de Rawls (1971) dans laquelle il décèle un archétype de la justice transcendantale. Cette prise de position a provoqué un débat intense. Gamel (2010), Kandil (2010), Valentini (2011), Satz (2012), Freeman (2012), Robeyns (2012), Ege et al. (2012), parmi d'autres, ont contesté l'opposition entre les deux types d'approche de la justice. Notre objectif n'est pas de revenir en détails sur ce débat. Nous le synthétisons afin de faire ressortir la complémentarité des deux approches ; ce qui finalement permet de légitimer l'approche comparative tout en la remettant à sa « juste » place par rapport à l'approche transcendantale.

Pour commencer nous rappelons d'abord la position de Sen à partir d'un exemple qu'il fournit. Nous résumons ensuite l'opposition à la dichotomie proposée par Sen.

Imaginons trois enfants qui se disputent pour l'attribution d'une flûte. Anne est la seule qui sait jouer de la flûte et pour cette raison revendique le droit de l'obtenir. Bob défend au contraire le fait qu'il est pauvre et qu'il n'a aucun jouet et devrait donc être le possesseur de la flûte. Enfin, Carla indique qu'elle a fabriqué cette flûte et donc qu'elle en est la légitime utilisatrice. Les trois arguments avancés par les enfants semblent légitimes. Tandis qu'une justice transcendantale ne pourrait pas traiter ce genre de situation liée au pluralisme des principes, la justice comparative aurait précisément pour objet de produire un ordonnancement, ne serait-ce que partiel, par classement par dominance. Cela ne signifie pas que la justice comparative produise un ordre complet. L'approche comparative fournit un ordre partiel, soit parce que nous jugeons une option à partir de points de vue conflictuels (comme dans l'exemple de la flûte), soit parce que nous n'avons pas assez d'information sur les options en présence pour les ordonnancer (comme c'est le cas souvent avec des options concernant notre futur), soit que certaines options ne puissent être comparées avec une métrique commune (comme c'est le cas de biens incommensurables) (Satz, 2012). «L'essentiel est d'obtenir un accord fonctionnel sur un ensemble de sujets facilement identifiables par l'injustice manifeste qu'ils reflètent » (Sen, 1999, p.333). Cet ordre partiel n'est pas le fruit d'une référence à un principe transcendantal mais de la confrontation des arguments de chacun lors de l'épreuve de la discussion publique (Sen, 1999, 2009). En somme, l'approche comparative d'une part ne vise qu'à établir un ordre partiel, d'autre part admet l'incomplétude. Des informations complémentaires ou un changement de contexte peuvent ainsi produire un changement d'ordonnancement.

Bien que la justice comparative présente un intérêt majeur, il serait excessif de l'opposer aux approches transcendantales. Les deux approches ne visent pas le même objectif. La démarche transcendantale consiste à justifier un système ou un principe donné d'évaluation, sans pour autant procéder à cette évaluation ; tandis que l'approche comparative vise à utiliser des outils qui permettent d'accomplir une évaluation (Kandil, 2010), sans par conséquent que les principes n'aient eu besoin d'être légitimés.

D'ailleurs Sen (2012) précise qu'il n'estime pas que l'approche transcendantale soit redondante ou inutile, mais simplement qu'elle n'est ni nécessaire ni suffisante pour établir un ordonnancement des jugements concernant la justice dans des cas précis. Nous pouvons certes admettre avec Sen que l'approche transcendantale n'est ni nécessaire ni suffisante pour établir un classement, mais il faut en contrepartie admettre que la robustesse de ce classement doit reposer sur une légitimité des critères utilisés, que ces critères soient raisonnables; ce qui renvoie in fine à une approche transcendantale. Comme le soulignent Ege et al. (2012), la notion «d'impartialité ouverte» que développe Sen, qui lui permet de fonder des comparaisons qui ne reposent pas sur des critères purement positionnels, renvoie à une interprétation du spectateur impartial d'Adam Smith fort présente chez Rawls. Valentini (2012) relève que Sen commet un excès en interprétant la théorie de la justice de Rawls comme un ensemble d'institutions idéales. Elle ne vise pas à établir un ensemble 
d'institutions parfaites, mais, sur la base de principes de justice, ouvre la voix à de multiples arrangements institutionnels qui rendront la société juste, en fonction de son contexte, en cohérence avec les principes de justice. Les principes de justice ne sont que des points de départ, pas des conclusions de la théorie de la justice. Ils laissent ouverts les arrangements sur une base comparative.

Nous pouvons par conséquent conclure qu'il faut distinguer les principes de justice des ordonnancements qui sont réalisés sur la base de principes. Ces principes méritent certainement d'être légitimés par des approches transcendantales, tandis que les approches comparatives peuvent apporter un éclairage, dans des cas concrets, d'ordonnancements que les populations établissent sur la base de critères auxquels elles se réfèrent. Or la justice environnementale s'appuie sur trois principes : l'équité, la reconnaissance et la participation. Nous ne discutons par de la pertinence de ces trois principes d'un point de vue transcendantal. Les insuffisances des théories distributives de la justice ont été discutées à différentes reprises notamment au regard de la portée du principe de reconnaissance (voir par exemple Fraser, 2000 ; Fraser et Honneth, 2003 ; Young, 1990). Nous nous concentrons sur une approche comparative en nous demandant en quoi l'approche par les capabilités défendue par Sen constitue une voie pertinente pour appréhender les enjeux de justice environnementale.

\section{Approche des capabilités et justice environnementale}

Nous avons recours à une approche comparative de la justice sur la base de l'approche des capabilités parce que cette dernière nous semble permettre d'appuyer la notion de justice environnementale avec quelques aménagements. Nous ne visons pas à défendre, ni à produire une théorie de la justice. Conformément à son approche comparative Sen (2009) souligne d'ailleurs que l'approche des capabilités ne vise pas fournir une théorie de la justice et que l'interpréter comme telle serait une erreur. En revanche, elle fournit «...une base informationnelle sur laquelle se concentrer pour juger et comparer les avantages globaux » $(2010, \text { p. } 285)^{4}$.

Les capabilités constituent une base informationnelle de justice plus large que le revenu ou l'utilité ou les biens premiers (à la Rawls). La justice n'est plus réduite à l'enjeu de la distribution des ressources rares mais inclut aussi la liberté de choix. L'enjeu n'est pas de définir une justice parfaite, mais de savoir comment procéder de façon juste dans notre vie quotidienne. Une approche comparative de la justice requiert simplement une évaluation de l'ensemble de capabilités des personnes ou des groupes sociaux et de sa progression. Elle se situe dans une idée de progrès vers la justice. Dans cette perspective, l'injustice peut être considérée comme une inégalité des capabilités dans le temps et dans l'espace (Ballet et al., 2013). L'approche des capabilités offre une base pour une justice comparative, sans qu'il ne soit nécessaire de se référer à une théorie de la justice. Or précisément, la question de la justice environnementale n'est pas posée sur la base d'une théorie de la justice mais à partir de cas concrets.

Nous ne rappellerons pas cette approche, désormais bien connue (voir Alkire, 2002 ; Gasper, 2007 ; Deneulin, 2006 ; Bonvin et Farvaque, 2008 ; Crocker, 2008 parmi d'autres). Nous nous limitons à souligner en quoi elle apporte un éclairage consistant sur la justice environnementale et peut constituer une métrique adéquate pour comparer les cas relatifs à la justice environnementale. Pour cela, nous appuierons les trois dimensions fondatrices de la justice environnementale : la distribution, la participation et la reconnaissance.

\footnotetext{
${ }^{4}$ Nous utilisons dans les passages qui suivent la référence à la traduction française parue en 2010 de Idea of Justice.
} 


\subsection{Approche par les capabilités et distribution des injustices environnementales}

Premièrement, elle assure une lecture des enjeux de distribution via les possibilités réelles dont disposent les individus. Pour citer Sen (2010, p.286), «L'approche par les capabilités se concentre sur la vie humaine et pas seulement sur des 'objets de confort' comme les revenus ou les produits de base- souvent érigés en critères principaux du succès humain, notamment dans l'analyse économique. Elle propose d'abandonner la focalisation sur les moyens d'existence pour s'intéresser aux possibilités réelles de vivre ». Le focus sur les possibilités réelles est essentiel dans la justice environnementale dans la mesure où les cas relatés soulignent à l'évidence des atteintes graves aux capabilités de base des individus telles la santé. Sen a ainsi proposé dans son ouvrage de 1980, Equality of What?, l'expression " capabilités de base" (basic capabilities) pour désigner la capacité de satisfaire des fonctionnements élémentaires et d'une importance cruciale tels que se nourrir, se loger, échapper aux maladies évitables et à la mort prématurée, etc. Nussbaum (1988) emploie également la notion de capabilités de base, mais cette expression désigne pour elle les capabilités potentielles qu'une personne pourrait développer et non pas un ensemble restreint de capabilités élémentaires. Elle propose néanmoins une liste de dix capacités fondamentales, sous-divisées en capacités secondaires, pouvant servir de référence universelle (Nussbaum 2000). Cependant, une telle liste ne revient pas à établir un classement. La valeur accordée aux différentes capabilités peut diverger selon le contexte, ou encore selon les individus. Pour cette raison, Sen entend conserver une certaine flexibilité dans la formulation de ce que sont les capabilités. Plutôt que de partir de la définition d'une liste universelle de capabilités de base, il opte pour la justification d'un ensemble de capabilités par une procédure de délibération démocratique ${ }^{5}$.

\subsection{Approche par les capabilités et enjeu de participation}

Deuxièmement, du fait de cette procédure de délibération démocratique, elle donne voix aux populations et rejoint la question de leur participation aux décisions. Sen insiste en effet sur le fait que les capabilités à prendre en compte sont celles que les individus ont des raisons de valoriser. Pour cette raison, l'approche des capabilités insiste sur la capacité à être agent de sa situation. Cette capacité à être agent découle bien sûr du degré de liberté que possède l'individu. Plus il sera libre d'agir, plus il sera en position d'être agent de sa vie. Les personnes qui n'exercent pas leur agencéité sont passives, soumises aux situations dans lesquelles elles se trouvent, ou sous l'influence des autres. Selon Alkire (2008), l'agencéité recouvre cinq caractéristiques. a) Elle renvoie aux buts et objectifs que vise l'agent. Pour chaque but et objectif, l'agent possède une capacité d'agence plus ou moins forte. Mais de plus, à un but ou objectif peuvent correspondre plusieurs réalisations. Les diverses réalisations ne sont pas toujours parfaitement compatibles et la capacité d'agence d'un individu se reflètera aussi dans sa capacité à aménager les conditions de faisabilité des diverses réalisations. b) Elle inclut à la fois le pouvoir effectif (effective power) de la personne ou du groupe de personnes à atteindre ses objectifs et le contrôle sur la réalisation des objectifs. La notion de contrôle renvoie à la capacité de la personne à assumer des choix et à contrôler la procédure de réalisation des choix directement, qu'elle aboutisse ou non à la réalisation voulue. En revanche, le pouvoir effectif reflète la capacité d'aboutissement, que la personne contrôle directement ou non le choix et la procédure de réalisation. Le pouvoir effectif suppose donc la prise en compte des interactions sociales dans la mesure où la réalisation

\footnotetext{
${ }^{5}$ D'un point de vue pragmatique, plusieurs procédures destinées à établir un ensemble ou une liste de capabilités de base sont possibles. Alkire (2002) propose une discussion éclairée des alternatives et des questions et enjeux qui y sont associés.
} 
effective d'un fonctionnement peut dépendre de l'action des autres. c) Elle est relative à la capacité à réaliser son bien-être mais aussi celui d'autres agents. Ainsi, réduire la torture ou vaincre la faim dans le monde peuvent être des objectifs auxquels la personne accorde de la valeur. Sa capacité d'agence est dans ce cas aussi relative à sa capacité d'exercer un contrôle direct sur cet objectif et/ou à avoir un pouvoir effectif à atteindre ces objectifs. Elle ne concerne pas seulement le bien-être de la personne, mais l'ensemble des objectifs que cette dernière se fixe. Certains de ces objectifs peuvent viser le bien-être d'autres personnes, des animaux $^{6}$, etc. d) Elle est associée aux objectifs auxquels la personne accorde de la valeur, ce qui signifie que tout ce qui est réalisé au moins partiellement par la personne ne peut être attribué systématiquement à sa liberté d'agence. Il faut que la personne accorde une valeur à cette réalisation. e) La capacité d'agence implique la responsabilité dans la réalisation ou l'état des choses. Puisque l'agent choisit librement, il est de fait responsable de ses choix.

Nous pouvons considérer que la participation passe par la valorisation de la capacité d'agence. Cette capacité d'agence suppose les trois critères de participation discutés dans les mouvements pour la justice environnementale : la mise à disposition de l'information sans laquelle l'individu ne peut établir un choix raisonné ; être partie prenante sans quoi il ne peut exprimer les raisons qu'il a de valoriser tel ou tel objectif et ne peut contrôler la procédure qui permet les choix ; la coproduction d'information scientifique qui renvoie à la fois au fait de contrôler la procédure mais aussi au pouvoir effectif d'atteindre les objectifs. Cependant, accorder une considération essentielle à la justice dans le processus de participation, comme le revendiquent les mouvements en faveur de la justice environnementale, suppose de donner une priorité à la justice dans le processus de choix sur la justice dans les choix eux-mêmes. Une telle position renverse les priorités proposées dans l'approche des capabilités par Sen. En effet, Sen $(2004 b, 2005)$ distingue deux conceptions de la justice : d'une part la liberté d'opportunités, d'autre part la liberté de processus. Il illustre cette distinction par l'exemple d'une jeune fille, Natacha. Si nous supposons que Natacha est contrainte de sortir le soir par une autorité (par exemple ses parents), elle n'est donc pas libre de choisir de sortir ou non. Même si elle désire sortir et que la contrainte correspond de fait avec son désir, elle n'est pas libre pour autant. Cette situation implique deux problèmes : a) Natacha n'a pas son mot à dire dans la décision la concernant, b) elle n'a pas d'autres opportunités que de sortir. Selon Sen, l'approche des capabilités se focalise avant tout sur l'absence d'opportunités alternatives. Or ce que soulignent bien les mouvements en faveur de la justice environnementale est que l'absence d'opportunités alternatives découle fondamentalement d'une absence de participation. La justice dans le processus de décision est prioritaire sur la justice dans les opportunités puisque la première conditionne les alternatives de la seconde. L'approche des capabilités peut par conséquent constituer une contribution significative à la justice environnementale sous condition qu'elle admette une inversion des priorités dans les deux types de libertés.

\subsection{Approche par les capabilités et reconnaissance}

Troisièmement, l'approche des capabilités constitue une approche cohérente avec la nécessité de reconnaissance des populations. Cela suppose néanmoins une discussion sur la notion de capabilités collectives. Selon Evans (2002) la capacité d'agence est façonnée par les valeurs communes et les structures sociales. Ce n'est pas parce que chacun possède des droits qu'il s'engage automatiquement avec et envers les autres. Au contraire, en tant que personne responsable, mon engagement prend valeur parmi toute une série d'interactions sociales qui

\footnotetext{
${ }^{6}$ Le bien être des animaux, (dans une perspective utilitariste) se réduit souvent à la prise en compte de la souffrance. $C f$. Porphyre dans sa réédition de 2003 et plus récemment Singer (1993). Cf. en contre point, Nussbaum (2006).
} 
donnent un sens à cette responsabilité (Ballet et al., 2007). Une attention particulière doit donc être accordée au rôle du débat public, aux espaces de discussions et aux possibilités d'interactions sociales itératives pour l'émergence de valeurs et d'engagements communs. Les capabilités collectives émergent de ces interactions sociales et sont différentes de la somme des capabilités individuelles. Elles permettent d'atteindre des objectifs que les capabilités individuelles ne garantissent pas de réaliser. La capacité d'agence collective ou la capacité d'un groupe à agir ne vise pas seulement à changer le niveau de bien-être de ses membres, mais aussi à favoriser le changement dans la société (Ibrahim, 2008).

Sen (2010) a récemment clarifié sa position sur les capabilités collectives. Il reconnaît d'une part, les influences sociales sur les individus : «Il est difficile d'imaginer que des personnes vivant en société puissent penser, choisir ou agir sans être influencées par la nature et le fonctionnement du monde qui les entoure. » (p.299). Il admet d'autre part, la notion de capabilité collective : "Il n'y a, bien sûr, aucune difficulté majeure à penser des capabilités de groupe. Lorsque nous disons, par exemple, que l'Australie peut vaincre dans les matchs d'essai tout autre pays qui pratique le cricket,..., nous parlons de la capabilité de l'équipe australienne de cricket et non de tel ou tel joueur australien en particulier.» (p.298). En revanche, il réduit la portée des capabilités collectives dans les jugements d'évaluation de la société et donc leur rôle dans la justice comparative. S' «il n'a aucune raison particulière relevant de l'analyse d'exclure a priori les capabilités collectives... Les arguments qui dissuadent de prendre ce chemin portent sur la nature du raisonnement que cela impliquerait » (p.300). «Puisqu'un groupe ne 'pense' pas dans le sens évident où le font les individus, l'importance de ses capabilités collectives serait plus ou moins comprises, pour des raisons assez claires, en fonction de la valeur que leur accordent ses membres... En dernière analyse, c'est sur des évaluations individuelles qu'il nous faudrait prendre appui, tout en reconnaissant l'interdépendance profonde des jugements d'individus qui interagissent» (p.300). Sen distingue en fait deux niveaux, celui de la capacité d'action ou agencéité qui peut tout à fait prendre en compte les capabilités collectives ou les capacités d'agence collectives et celui du jugement de l'évaluation sur la société qui permet la réalisation d'une approche comparative de la justice. Or à l'inverse du premier niveau qui admet la capabilité collective, le second niveau doit s'appuyer uniquement sur les capabilités individuelles. La distinction qu'opère Sen serait robuste seulement si les capabilités collectives en tant que moyens d'action produisaient des effets sur les capabilités individuelles uniquement et que l'on pouvait réduire tous ces effets à des capabilités individuelles. Or ce qu'illustrent parfaitement les mouvements pour la justice environnementale est que le cœur des revendications porte sur la reconnaissance. Il ne s'agit pas de reconnaissance individuelle, mais d'identité collective. La reconnaissance des spécificités des groupes n'est pas simplement un moyen de renforcer leur capacité d'action au profit des individus pris séparément, elle est aussi un enjeu de justice comparative et donc d'évaluation de la société. Il s'agit de reconnaître une identité collective et une culture. Et lorsque Sen (2010) en défense de sa position note que «Les thèses qui réduisent une personne au seul statut de membre d'un groupe unique sont généralement fondées sur une interprétation inadéquate de l'envergure et de la complexité de toute société dans le monde » (p.302), il commet l'erreur de considérer qu'octroyer une identité de groupe équivaut à réduire l'identité individuelle à cette identité de groupe. Il est vrai que Sen s'est prononcé à plusieurs reprises sur la notion d'identité et appuie l'idée de pluralité de l'identité (par exemple Sen, 2004a). Or pourquoi le fait de se reconnaître dans une identité de groupe reviendrait à éliminer les autres identités ? Il s'agit là d'une position de principe qui vise à contrer le recours aux identités collectives comme source de violence (Sen, 2006b), mais qui est sans fondements. Ne pas reconnaître l'identité collective de certains groupes qui réclament cette reconnaissance est aussi une forme de violence. Ce que soulignent avec force les mouvements pour la justice environnementale. Une justice comparative doit tenir compte 
dans son évaluation de la reconnaissance des identités collectives. Or pour cela, une évaluation qui se limiterait à comparer des capabilités individuelles laisserait de côté une dimension fondamentale de la justice environnementale. Il n'y a aucun sens à reconnaître une caractéristique de l'identité d'une personne sans en même temps reconnaître celle du groupe. Et ce n'est pas en évaluant les capabilités individuelles sur l'identité que nous pouvons établir la reconnaissance d'une identité de groupe. C'est précisément le contraire qui s'opère. La reconnaissance des identités collectives constitue une extension des capabilités des individus. Dans l'évaluation d'une société, il convient alors d'attribuer un poids à la reconnaissance des identités collectives. Ce qui ne revient certainement pas à réduire l'identité des personnes à ce trait de leur identité. Mais permet en revanche de reconnaître leur capacité d'agence collective.

Comme le fait remarquer Honneth (2010), si le mépris est ressenti par les individus, il n'est pas seulement individuel. Il concerne également les modes de vie jugés inférieurs ou imparfaits. L'absence de reconnaissance de certaines valeurs collectives affecte directement les individus qui les portent au point que leurs existences n'ont plus aucune signification positive. L'injustice du mépris est le pendant négatif de la reconnaissance. Une société qui vise à réduire les injustices et qui évalue comparativement ses progrès en matière de justice, ne peut éluder les progrès dans la reconnaissance des cultures et des modes de vie. Une approche comparative de la justice qui s'appuierait sur les capabilités ne peut se passer de tenir compte des capabilités collectives. La position prise par Sen (2009) sur les capabilités collectives nous parait, pour cette raison, intenable. Si la justice comparative a pour objet, comme l'affirme Sen, d'identifier les moyens qui réduiront les injustices, les capabilités collectives constituent un objet d'évaluation sociale indispensable. La capacité d'agence collective est la base de la reconnaissance.

\section{Conclusion}

Dans cet article nous avons défendu que l'approche par les capabilités offre de sérieuses pistes pour donner tout son sens à la justice environnementale abordée sous l'angle de la justice comparative. Les personnes défavorisées vivent souvent dans un environnement de faible qualité comparativement aux autres et l'approche des capabilités peut constituer une métrique adéquate pour évaluer leur situation.

Cependant, deux ajustements de cette approche sont nécessaires. Tout d'abord, elle doit mettre au centre de son analyse la justice dans le processus et ce de manière prioritaire sur la justice dans les opportunités. Ensuite, elle doit tenir compte des capabilités collectives, pas uniquement comme élément de la capacité d'agence des individus, mais également et fondamentalement dans l'évaluation sociale des sociétés. Ces deux ajustements appellent par conséquent à un dépassement de l'approche des capabilités telle que conçue par Sen.

\section{Références}

Agyeman, J., Bullard, R.D., Evans B. (eds) 2003. Just Sustainabilities: Development in an Unequal World. Cambridge, MA: MIT Press.

Alkire, S. 2008. 'Concepts and Measures of Agency', Working paper Nº9, OPHI Working Paper Series, www.ophi.org.uk.

Alkire, S. 2002. Valuing Freedoms. Sen's Capability Approach and Poverty Reduction. Oxford: Oxford University Press.

Ballet, J., Dubois, J.-L. and Mahieu, F.-R. 2007. 'Responsibility for Each Other's Freedom: Agency as the Source of Collective Capability', Journal of Human Development, 8(2):185201. 
Ballet, J., Koffi, J-M., Pelenc, J. 2013. 'Environment, Justice and the Capability Approach'. Ecological Economics, 85(1): 28-34.

Bonvin, J.-M., Farvaque E. 2008. Amartya Sen. Une politique de la liberté. Paris: Michalon.

Bretting, J.G., Prindeville D.-M. 1998. 'Environmental Justice and the Role of Indigenous Women organzing Their Communities', in D. Camacho (ed.), Environmental Injustices, Political Struggles: Race, Class, and the Environment: 141-164, Durham, NC: Duke University Press.

Bryant, B., Mohai, P. (eds) 1982. Race and the Incidence of Environmental Hazards: A Time for Discourse. Boulder, CO: Westview Press.

Bullard, R. 1993. Confronting Environmental Racism: Voices from Grassroots. Boston, MA: South End Press.

Crocker, D. 2008. Ethics of Global Development. Agency, capability, and Deliberative Democracy, Cambridge: Cambridge University Press.

Deneulin, S. 2006. The Capability approach and the praxis of development, Basingstoke: Palgrave MacMillan.

Di Chiro, G. 1992. 'Defining Environmental Justice: Women's Voices and Grassroots Politics'. Socialist Review, 22(4): 93-131.

Edwards, B. 1995. 'With Liberty and Environmental Justice for All: Te Emergence and Challenge of Grassroots Environmentalism in the United States', in B. R. Taylor (ed.), Ecological Resistance Movements. The Global Emergence of Radical and Popular Environmentalism: 35-55, Albany, NY: Suny Press.

Ege R., Igersheim H., Le Chapelain C. 2012. 'Transcendental vs. Comparative Approaches to Justice: A Reappraisal of Sen's Dichotomy', Working paper BETA, Université de Strasbourg, n²012-270.

Epstein, B. 1997. 'The Environmental Justice/Toxics Movement: Politics of Race and Gender', Capitalism, Nature, Socialism, 8(3): 63-87.

Evans, P. 2002. 'Collective Capabilities, Culture and Amartya Sen's Development as Freedom', Studies in Comparative International Development, 37(2): 54-60.

Faber D.R., McCarthy, D. 2003. 'Neo-Liberalism, Globalization, and the Struggle for Ecological Democracy: Linking Sustainability and Environmental Justice', in Agyeman J., Bullard R.D. et Evans B. (eds), Just Sustainabilities: Development in an Unequal World: 38-63, Cambridge, MA: MIT Press.

Fraser, N. 2000. 'Rethinking Recognition', New Left Review, 3: 107-120.

Fraser, N. et Honneth, A. 2003. Redistribution or Recognition? A Political-Philosophical Exchange, Londres: Verso.

Freeman, S. 2012. 'Ideal Theory and the Justice of Institution vs. Comprehensive Outcomes', Rutgers Law Journal, 43: 169-209.

Freudenberg, N., Steinsapir, C. 1992. 'Not in Our Backyards: The Grassroots Environmental Movement', in R. E. Dunlap et A.G. Mertig (eds), American Environmentalism: The U.S. Environmental Movement, 1970-1990: 27-35, Philadelphia, PA: Taylor \& Francis.

Gamel, C. 2010. 'Que faire de l'approche par les capacités? Pour une lecture rawlsienne de l'apport de Sen', Working paper GREQAM, Université d'Aix-Marseille, n²010-30.

Gasper, D. 2007. 'What is the Capability Approach? Its Core, Rational, Partners, and Dangers', Journal of Socio-Economics, 36(3): 335-359.

Getches, D.H., Pellow, D.N. 2002. 'Beyond "Traditional" Environmental Justice', in K.M. Mutz; G.C. Bryner et D.S. Kenney (eds), Justice and Natural Resources: Concepts Strategies, and Applications: 3-30, Washington, DC: Island Press.

Gibbs, L. 1982. Love Canal: My Story. Albany, NY: Suny Press. 
Hamilton, C. 1994. 'Concerned Citizens of South Central Los Angeles', in R. Bullard (ed.), Unequal Protection: Environmental Justice and Communities of Color: 207-219, San Fransisco, CA: Sierra Club Books.

Honneth, A. 2000. La lutte pour la reconnaissance, Paris : Gallimard.

Ibrahim, S. 2008. 'Collective Agency: Wider Freedoms and New Capabilities Through Selfhelp': 61-81, in J.-L. Dubois, A.-S. Brouillet, P. Bakhshi and C. Duray-Soundron (Eds) Repenser l'action collective: Une approche par les capabilities, Réseau Impact, Paris : L'Harmattan.

Kandil, F. 2010. 'Idéal ou comparative: Quelle approche pour la justice sociale ?'. Revue économique, 61 : 213-236.

Krauss, C. 1994. 'Women and of Color on the Front Line': 256-271, in R. Bullard (ed.), Unequal Protection: Environmental Justice and Communities of Color, San Fransisco, CA: Sierra Club Books.

LaDuke, W. 2002. The Winona LaDuke Reader. Stillwater, MN: Voyageur Press.

Leopold, A. 1949. 'A Sand County Almanac and Sketches Here and There'. Oxford University Press: Oxford.

Naess, A. 1973. 'The Shallow and the Deep, Long-Ranged Ecology Movement: a Summary'. Inquiry 16 (1): 95-100.

Melosi, A. 2004. Garbage in the Cities: Refuse, Reform, and the Environment. Pittsburgh, PA: University of Pittsburgh Press.

Nussbaum, M.C. 2000. Women and Human Development: The Capabilities Approach, Cambridge: Cambridge University Press.

Nussbaum, M. 2006. Frontiers of Justice, Cambridge: Harvard University Press.

Nussbaum, M.C. 1988. 'Nature, Function and Capability. Aristotle on Political Distribution': 145-184, in J. Annas (ed.) Oxford Studies in Ancient Philosophy, supplementary volume.

Passmore, J.A. (1974). Man's Responsibility for Nature: Ecological Problems and Western Traditions. Duckworth: London.

Pellow, D.N. 2000. 'Environmental Inequality Formation: Toward a Theory of Environmental Injustice'. American Behavioral Scientist, 43(3): 581-601.

Porphyre 2003. De l'abstinence, Paris : Les belles Lettres, III, para.3:19-20.

Prindeville, D.-M. 2004. 'The Role of Gender, Race/Ethnicity, and Class in Activists' Perceptions of Envrionemental Justice': 93-108, in R. Stein (ed.), New Perspectives on Environmental Justice: Gender, Sexuality, and Activism, New Brunswick, NJ: Rutgers University Press.

Pulido, L. 1996. Environmentalism and Social Justice: Two Chicano Struggles in the Southwest. Tucson, AZ: University of Arizona Press.

Rawls, J. 1971. A Theory of Justice. Cambridge, MA: Harvard University Press.

Robeyns, I. 2012. 'Are Transcendental Theories of Justice Redundant?', Journal of Economic Methodology, 19: 153-163.

Rolston, H. 1975. 'Is There an Ecological Ethic? Ethics': An International Journal of Social and Political Philosophy, 85: 93-109.

Satz, D. 2012. 'Amartya Sen's The Idea of Justice: What Approach, Which Capabilities?' Rutdgers Law Journal, 43: 277-293.

Schlosberg, D. 2007. Defining Environmental Justice. Theories, Movements, and Nature, Oxford: Oxford University Press.

Schlosberg, D. 2003. 'The Justice of Environmental Justice: Reconciling Equity, Recognition, and Participation in a Political Movement': 77-106, in A. Light et A. De-Shalit (eds), Moral and Political Reasoning in Environmental Practice, Cambridge, MA: MIT Press.

Schlosberg, D. 1999. Environmental Justice and the New Pluralism: The Challenge of Difference for Environmentalism. Oxford: Oxford University Press. 
Sen, A.K. 2012. 'Symposium on the Idea of Justice. Rutgers University. A Reply'. Rutgers Law Journal, 43: 317-335.

Sen, A.K. 2009. Idea of Justice, London, Penguin Books, traduction 2010, L'idée de justice, Paris: Flammarion.

Sen, A.K. 2006a. 'What Do We Want From a Theory of Justice?'. Journal of Philosophy CII(5): 215-238.

Sen A.K. 2006b. Identity and Violence: the Illusion of Destiny, New York: W. W. Norton \& Co.

Sen, A.K. 2005. 'Human Rights and Capabilities'. Journal of Human Development, 6(2): 151166.

Sen, A.K. 2004a. 'Social Identity'. Revue de Philosophie Economique, 9: 7-27.

Sen, A.K. 2004b. 'Elements of a Theory of Human Rights'. Philosophy and Public Affairs, 32(4): 315-356.

Sen, A.K. 1980. Equality of What?, Tanner Lectures on Human Values, Cambridge: Cambridge University Press.

Singer, P. 1993. La libération animale, Paris : Grasset.

Stone, C.D. 1974. Should Trees Have Standing? Toward Legal Rights for Natural Objects. Los Altos, CA: W. Kaufmann.

Taylor, D.E. 2000. 'Environmentalism and the Politics of Exclusion': 53-62, in Bullard R.D. (ed.), Confronting Environmental Racism: Voices from the Grassroots, Boston, MA: South End Press.

Valentini, L. 2011. 'A Paradigm Shift in Theorizing About Justice? A Critique of Sen'. Economics and Philosophy, 27: 297-315.

Wapner, P., Matthew, R.A. 2009. 'The Humanity of Global Environmental Ethics'. Journal of Environment and Development, 18 (2), 203-222.

Young, I. 1990. Justice and the Politics of Difference, Princeton, NJ.: Princeton University Press. 